\title{
Desmopressin reduces risk of bleeding after percutaneous kidney biopsy
}

A phase IV study has shown that treatment with desmopressin acetate decreases the risk of bleeding after a percutaneous kidney biopsy and reduces hematoma size. "To our knowledge, this is the first randomized controlled trial that shows the efficacy of desmopressin in percutaneous kidney biopsy to decrease bleeding complications in patients without coagulation disorders," says investigator Carlo Manno.

Desmopressin is a synthetic derivative of vasopressin that exerts its hemostatic effects by increasing levels of von Willebrand factor and factor VIII. To evaluate the efficacy of desmopressin, 162 patients with recurrent macrohematuria and/or urinary abnormalities or nephrotic syndrome were randomly assigned to receive desmopressin $(n=80)$ or to receive placebo $(n=82) 1 \mathrm{~h}$ before a native kidney biopsy. Baseline characteristics were similar in the two groups. In all patients, ultrasound was performed immediately before the biopsy and $24 \mathrm{~h}$ after the biopsy. The primary outcome measure was the incidence of bleeding complications after the biopsy procedure. Secondary outcome measures included hematoma size, postbiopsy hemoglobin level, glomerular filtration rate, and length of hospital stay.

\section{4 ... bleeding was significantly less common in desmopressin- treated patients... 77}

Postbiopsy bleeding was significantly less common in desmopressin-treated patients than in placebo-treated patients ( $13.7 \%$ versus $30.5 \%$, respectively). Among the 36 patients who experienced bleeding, median hematoma size was $208 \mathrm{~mm}^{2}$ versus $380 \mathrm{~mm}^{2}$ in patients receiving desmopressin and placebo, respectively. Mean hospital stay was 1 day less in the desmopressin group than in the control group, which led to considerable cost savings. No other differences were found between the groups in terms of secondary outcome measures. The treatment caused no serious adverse events, and had only mild side effects in three patients (a transient and small increase in heart rate). "Desmopressin is already known to be useful in patients at risk of bleeding and our results confirm its role in the prevention of bleeding complications even in patients not at risk," comments Manno. "For patient care, it would be useful to recommend the inclusion of such prophylaxis in the guidelines for renal biopsy."

Helene Myrvang

Original article Manno, C. et al. Desmopressin acetate in percutaneous ultrasound-guided kidney biopsy: a randomized controlled trial. Am. J. Kidney Dis. doi:10.1053/j.ajkd.2010.12.019 